\title{
AMENDMENTS
}

\section{Publisher Correction: Translational neuroscience applications for automated detection of rodent grooming with deep learning}

Nicholas J. Burton, Léonie Borne (D) and Elizbeth E. Manning

Correction to: Lab Animal https://doi.org/10.1038/s41684-021-00830-y, published online 13 August 2021.

In the version of this News \& Views initially published, the accent was missing in the second author Léonie Borne's name. A reference omission (ref. 10, Aldridge, J. W., Berridge, K. C. \& Rosen, A. R. Can J Physiol Pharmacol 82, 732_739, https://doi.org/10.1139/y04-061 (2004)) has now been included, with original ref. 10 becoming 11 (ref. 11, Berridge, K. et al. (2005)) and original ref. 11 becoming 12 (ref. 12, Hsu, A.I. et al. (2019)). In the final paragraph of main text, ref. callouts have thus been renumbered as follows: "More detailed analysis at this level of resolution has been used to highlight neural circuit control of grooming ${ }^{10}$, and there is evidence that microstructure detail may be necessary to characterize grooming changes relevant to behavioural disturbances in human brain disorders ${ }^{11}$.... Furthermore, studies of pathological grooming may benefit from unsupervised approaches to detect short-duration grooming abnormalities that may be difficult for human observers to detect ${ }^{12}$." Further, ref. 4 has been updated with series name (Advances in Neural Information Processing Systems) and url (http://papers.nips.cc/paper/4824-imagenet-classification-with-deep-convolutional-neural-networks.pdf).

These changes have been made to the online version of this News \& Views.

Published online: 16 September 2021

https://doi.org/10.1038/s41684-021-00870-4

๑ Springer Nature America, Inc. 2021

\section{Publisher Correction: Residents and regulations-always an adventure}

Liana A. Galex and Steven Shipley

Correction to: Lab Animal https://doi.org/10.1038/s41684-020-0619-9, published online 20 August 2020.

In the version of this Protocol Review initially published, an error appeared in the first author's name. The name was shown as "Llana A. Galex" instead of "Liana A. Galex."

The error has been corrected in the online version of the article.

Published online: 23 September 2021

https://doi.org/10.1038/s41684-021-00877-x

(c) Springer Nature America, Inc. 2021 